\title{
Is syncope a risk predictor in the general population?
}

\author{
Martin H. Ruwald ${ }^{1,2}$, Louise R. Olde Nordkamp ${ }^{3}$ \\ ${ }^{1}$ Department of Cardiology, Gentofte Hospital, Hellerup, Denmark \\ ${ }^{2}$ Heart Research Follow-up Program, Division of Cardiology, \\ University of Rochester Medical Center, Rochester, NY, United States \\ ${ }^{3}$ Heart Center, Department of Clinical and Experimental Cardiology, \\ Academic Medical Center, Amsterdam, the Netherlands
}

\begin{abstract}
Syncope in the general population is a frequent event often leading to hospitalization, but it is unclear whether syncope in the general population is an independent risk marker for adverse prognosis. In this review, we investigate the current literature and evaluate the prognosis and impact of syncope on adverse outcomes including death and recurrences across different populations with focus on the general population. In wide terms, a syncopal event is related to a higher risk of subsequent falls and injury and cardiac syncope is particularly associated with increased mortality as compared to non-cardiac syncope. The overall prognosis in the general population is by large determined by the underlying presence and severity of a given cardiac disease, but a given underlying cardiac disease can very well be unknown at the time of first syncope so that syncope is the presenting symptom resulting in an independent risk increase. Moreover, syncope is a significant risk predictor of a recurrence across populations. It is important to recognize several risk factors associated with adverse outcome in order to safely navigate in a population where most patients with syncope are healthy and low-risk but where a small number of patients have life-threatening conditions. Further research in the general population should attempt to categorize which patients with syncope need immediate referral and diagnostic testing, and whether this affects the outcome. (Cardiol J 2014; 21, 6: 631-636)
\end{abstract}

Key words: syncope, general population, prognosis, risk, cardiac syncope

\section{Introduction}

Assessment of risk for serious cardiovascular (CV) outcome after syncope is difficult. Confounding is one of the many challenges. Confounding in epidemiological studies of syncope particularly concerns the fact that, in general, patients with syncope are sicker than patients without syncope. Even though statistical analysis in studies may adjust for this imbalance of known influencing factors, other unknown factors may influence the results. This apparent issue, in particular, makes it difficult to evaluate if syncope per se is a risk factor in the general population. In order to further investigate this enigma, it is helpful to conceptualize the available data.

To understand if syncope is a risk predictor in various populations it is important first to establish which outcomes are relevant to consider for syncope patients. Among all syncope patients, a risk outcome could be traumatic fall-related injury because of a new syncopal episode (recurrent

Address for correspondence: Martin H. Ruwald, MD, PhD, Heart Research Follow-up Program, Division of Cardiology, University of Rochester Medical Center, 265 Crittenden Boulevard, Rochester, 14642 NY, USA, e-mail: mruwald@hotmail.com Received: 07.09.2014

Accepted: 23.09.2014 
syncope). Other outcomes to comprehend could be all-cause death, CV death or sudden cardiac death alone. Among younger patients, an outcome of interest could be future development of significant CV disease (CVD) and among elder patients, an outcome could be immediate or future need for pacemaker. Physicians must incorporate all one of these issues when evaluating a patient with syncope even if the episode most likely is a single case of vasovagal syncope.

Based on the above premises it is useful to look at specific age groups because of the variety in prevalence of important comorbidities, use of concomitant medications and, perhaps most importantly, the presumptive cause of the syncopal episode. Essentially, the question comes down to whether syncope in itself is a risk factor for mortality or if it is the underlying heart disease (known or unknown) that is the cause of a higher risk.

\section{Cause of syncope according to age and clinical setting}

The prevalence and causes of syncope differ depending on both age and the clinical setting in which the patient presents and is investigated. Additionally, differences in diagnostic definitions, administrative care pathways, and population demographics make comparison between different studies challenging [1-6]. Reflex (vasovagal) syncope, defined as a transient loss of consciousness caused by systemic arterial hypotension resulting from reflex vasodilatation or bradycardia or both, is the most frequent observed cause of syncope in all age groups. Syncope caused by various CVD is the second most observed cause. The prevalence of CVD however, varies significantly with clinical setting exhibiting higher frequencies among the elderly and in the emergency and cardiology settings. However, at least 2 issues pertain to these observations. First, the diagnostic accuracy of syncope testing and by basing specific diagnosis on the history (i.e. assigning the diagnosis based on typical vasovagal symptoms) differ between physicians, setting and patients. Second, the diagnosis of cardiac syncope or syncope related to CVD depends on the work-up, which may be inaccurate and differ by setting, physicians and patient preferences. In contrast to the relatively high frequency of concomitant CVD in the elderly seen in the hospital setting, cardiac origin of syncope is quite rare in the general population and in the young. This notwithstanding, overuse and a "shot-gun" approach of diagnostic procedures to diagnose (or fear of missing) a potential very rare and life-threatening channelopathy are common $[7,8]$.

Orthostatic hypotension is very frequent among old patients and a rare cause of syncope in the young. The rate of unexplained syncope similarly reflects the clinical setting with decreasing rate in specialized syncope clinics and cardiology settings. In all settings, the incidence of syncope increases with age with bimodal peaks in adolescence and in old age. In short, the elderly accounts for the vast majority of patients represented in the general population, general practice, and Emergency Department (ED) and Cardiology Departments. Moreover, the temporal relationship may be an issue. If syncope occurred 20 years before death, one wonders if the 2 events are really related. These aspects are important to keep in mind when trying to answer the question whether syncope is a risk factor in the general population.

\section{Prognosis in the general population}

The Framingham Heart Study [9] $(\mathrm{n}=7,814)$ evaluated the incidence and prognosis among the participants in this study of the general population. Syncope occurred in 822 patients during a follow-up of 17 years (6.2 per 1,000 person-years). This low incidence is in sharp contrast with later studies reporting a life-time cumulative incidence of 35\% [10], which can be explained by the fact that in the Framingham study, only subjects who experienced syncope during the study were asked if they had also experienced syncope at a younger age.

In the Framingham study, a presumed vasovagal cause was observed in $21 \%$, a cardiac cause in $9 \%$, while for $37 \%$ the cause of syncope remained unknown or unexplained. In adjusted multivariate analysis, the all-cause mortality was significantly higher in patients with cardiac cause of syncope when compared to patients with syncope from other causes. In secondary analyses, the authors further stratified by presence of CVD and reported that cardiac syncope with or without known presence of CVD were associated with increased mortality risk (hazard ratio [HR] 2.01, 95\% confidence interval [CI] 1.48-2.73). The study also provided reassurance that vasovagal syncope with or without presence of known CVD was not associated with excess mortality (HR 1.08, 95\% CI 0.88-1.34).

One key point was that it is important to recognize that cardiac syncope can be a precursor of sudden death with a relative high mortality rate independent of the presence of known CVD (in some cases), but the study had major limitations 
concerning the diagnostic classification of syncope and collected clinical information. In particular, it can be noted that the patients generally had no comorbidities but if they did the cause for syncope even if it were vasovagal would potentially be ascribed to something else. The diagnostic procedures were not noted or systematic. Further the presence of syncope with CVD is not the same as syncope caused by CVD resulting in possible mis-adjudication of syncope in the study.

\section{Prognosis in other populations}

Earlier (pre-implantable cardioverter-defibrillator era) reports by Kapoor et al. [11, 12] from hospitalized patients in the 1990's established that cardiac syncope had higher mortality than non-cardiac syncope. One study [11] $(\mathrm{n}=433)$ found 5-year mortality to be significantly higher $(51 \%)$ in patients with cardiac cause of syncope when compared to patients with non-cardiac or unknown cause of syncope (24\%). The incidence of sudden cardiac death was significantly higher $(33 \%)$ in patients with cardiac cause of syncope when compared to non-cardiac (5\%) or unknown cause of syncope (9\%). However, the outcome after cardiac syncope was determined by the underlying heart disease rather than syncope itself, because underlying heart diseases were risk factors for mortality, regardless of whether the patient had syncope or not. This observation is in contrast to the studies described below.

Vanbrabant et al. [13] compared the outcome of syncope patients $(\mathrm{n}=2,785)$ from primary care and matched these with patients without syncope $(\mathrm{n}=13,909)$. A serious outcome was defined as the occurrence of a new $\mathrm{CV}$ event or serious injury and was reported in $12 \%$ of the syncope patients within 1 year. The predictors for serious outcome were increasing age, presence of $\mathrm{CV}$ comorbidity and CV risk factors. In adjusted analysis, they showed that syncope was an independent predictor for serious outcome (HR 3.99, 95\% CI 3.44-4.63), but this study did not incorporate the cause of syncope in the analysis.

Additionally, several studies have investigated the prognosis after syncope in ED [14-21]. In general, in ED there is a shift towards more serious cardiac causes and, in older subjects, towards more orthostatic hypotension compared to the general population. Reflex syncope, however, remains the most prevalent cause of syncope. These studies identified important predictors of adverse outcomes and short-term mortality among syncope patients. In the Evaluation of Guidelines, in Syncope Study (EGSYS) [19] the 1-year mortality rate of syncope in general was $5 \%$ and in the second EGSYS study [22] the 2-year mortality rate was $9 \%$. In the Osservatorio Epidemiologico sulla Sincope nel Lazio Study (OESIL) [15], the 1-year mortality was $12 \%$, in the Short-term Prognosis of Syncope (STePS) Study [21] 6\%, in the Risk stratification of Syncope in the Emergency Department (ROSE) Study [23] 7\%, in a study by Martin et al. [20] $15 \%$ and $7 \%$ in a recent retrospective registry study from EDs [24]. These studies represent a broad mix of all-cause syncope patients and the characteristics and mortality rates likely reflect the patients seen in most ED in the Western world. Additionally, these studies developed several risk scores and classifications, but unfortunately, with unsuccessful implementation and validation. A meta-analysis summarized the most important risk factors associated with adverse outcome to be palpitations preceding syncope, syncope during effort, history of heart failure or ischemic heart disease and clinical and laboratory evidence of bleeding [25]. Similar to the general population, in the ED, cardiac syncope was also associated with both higher short- and long-term occurrences of adverse events [26].

Finally, in hospitalized syncope patients, a large registry-based study [27] $(\mathrm{n}=37,017)$ suggested that syncope in patients without any prior history of comorbidities may constitute an increased risk of $\mathrm{CV}$ morbidity and mortality when compared to a general background population. Multivariable Cox regression analysis showed significantly higher all-cause mortality and CV hospitalizations across age groups when compared to the control subjects without syncope. Importantly, the study did not evaluate the specific temporal relationship from syncope to death and did not have information on electrocardiography (ECG), lab tests or history and it is reasonable to question whether these patients truly were healthy because they did not have a prior hospitalization. The findings in this study emphasize the importance of careful, timely and thorough initial evaluation of all patients with syncope and the need for diligent risk stratification is warranted. However, no data has yet proven that risk stratification can alter outcomes.

\section{Syncope work-up}

A history should be obtained and physical examination and ECG should be performed in all 
patients, with the initial goal of risk stratification and exclusion of an acute illness, such as myocardial infarction or pulmonary embolism, as the cause of syncope. The precise cause of syncope is identified during the initial evaluation in fewer than half of the patients [28], but the presence or absence of heart disease offers important information about the need for further evaluation and risk stratification [29].

Among patients with various forms of established heart disease the overall prognosis and mechanism of patients with syncope depends on the severity and type of underlying heart disease. For most heart diseases, syncope is an independent marker of adverse prognosis and sudden death compared to patients without syncope often representing a malignant brady- or tachyarrhythmia. Underlying heart disease or not, cardiac syncope is associated with adverse prognosis across all studied populations. The mechanism of syncope reflects the underlying heart disease with increasing likelihood of arrhythmic syncope in more severe heart diseases and increasing likelihood of reflex syncope in less severe heart disease. In selected heart diseases, syncope is further associated with increased mortality independently of the causal mechanism [30-33]. Even in the absence of a firm diagnosis of cardiac syncope, the presence of structural cardiac abnormalities or evidence of a primary electrical disorder is associated with a poor prognosis.

In contrast, a structurally normal heart with a normal ECG is associated with a benign etiology for syncope and a favorable prognosis but such a statement needs to be confirmed in larger data. Echocardiography and ECG were not considered in the Danish study on the risk of mortality in presumably healthy people with syncope where syncope was shown to be an independent predictor of death [27]. The absolute risk of death after syncope in this study was very low. High-risk cases of syncope have either structural or electrical cardiac anomalies. As these anomalies may be subtle, particularly in the general population, it is essential to identify potentially lethal causes of syncope by ECG as shown in Table 1. Conditions that are quite common in the average general population and often have syncope as a presenting symptom or as an associated symptom are shown in Table 2 . Table 3 shows the risk factors that are suggestive of cardiac syncope or adverse outcome requiring prompt further investigations.
Table 1. Specific electrocardiography (ECG) features.

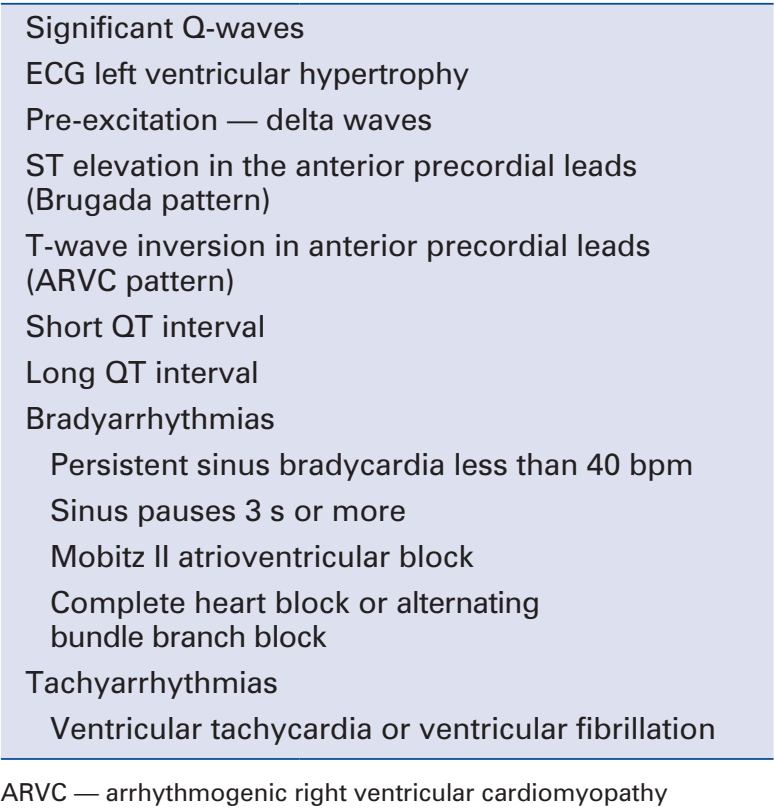

Table 2. Frequent cardiac conditions in the general population that often cause syncope.

\begin{tabular}{ll}
\hline Category & Specific condition \\
\hline $\begin{array}{l}\text { Valvular heart disease } \\
\text { Coronary artery disease } \\
\text { and thrombosis }\end{array}$ & $\begin{array}{l}\text { Acrtic stenosis } \\
\text { Acute ischemia or } \\
\text { myocardial infarction } \\
\text { Pulmonary embolism }\end{array}$ \\
Cardiomyopathy & $\begin{array}{l}\text { Ischemic heart failure } \\
\text { Non ischemic heart failure }\end{array}$ \\
$\begin{array}{l}\text { Cardiac conduction } \\
\text { disease }\end{array}$ & Sinus node dysfunction \\
\hline
\end{tabular}

\section{Risk of recurrence}

The risk of recurrent syncope remains an important issue when evaluating syncope as a risk predictor. It is well known that the number of previous syncopal episodes denotes the likelihood of another syncopal episode. In the general population, recurrent syncope occurs in approximately one third of the patients [8] and, although probably benign in origin, even vasovagal episodes are expected to result in increased traumatic fall-related injuries, work-related injuries, and motor vehicle accidents [34-36]. Increased susceptibility to syncope with advancing age is caused by age-related declining physiological regulation of heart 
Table 3. Risk factors suggestive of cardiac syncope or adverse outcome. Adapted from [8].

History
Palpitations at time of syncope
Shortness of breath
Syncope during exertion or in supine position
A family history of sudden cardiac death or
premature death
Significant heart disease
Prior myocardial infarction or other severe
coronary artery disease
Cardiomyopathy
Other significant structural heart disease
Comorbidities associated with syncope and
adverse outcomes
Renal failure
Severe anemia
Electrolyte disturbances
Other suggested features of adverse outcome
in syncope
Age > 65
High B-type natriuretic peptide levels
Low blood pressure at presentation

rate, blood pressure and in the cerebral blood flow. The risk of recurrence, particularly among the elderly, is additionally modified and increased by the number of $\mathrm{CV}$ drugs taken with orthostatic hypotension as side effect as well as influence of several comorbidities.

Recent data [37] from a large registry of hospitalized syncope patients $(\mathrm{n}=70,819)$ confirmed that a recurrence of syncope requiring hospital contact was associated with more than a 3 -fold increase in 1-year all-cause mortality and almost a 4-fold increase in 30-day CV death. Recurrent syncope was independently associated with increased risk of death signifying that recurrent syncope, concomitant CVD present or not, is a risk factor for mortality. Future studies from these databases will elucidate the incidence and influence of fall-related injuries and fractures as well as risk of motor vehicle accidents in this population. The Irish Longitudinal on Ageing (TILDA) [38] study is a population-based $(\mathrm{n}=8,163)$ project of adults over the age of 50 and was described in a recent review. Preliminary data from the study suggested that those with syncope were more likely to be female, have CVD or cerebrovascular disease, and be on $\mathrm{CV}$ and/or psychotropic medications. A total of $38 \%$ of patients had further experienced one or more falls in past year compared to $18 \%$ of those without syncope, signifying the overlap of symptoms and classification between falls and syncope - an issue that most likely underestimate the true incidence of syncopal attacks. Among the elderly, orthostatic intolerance syndromes comprise a heterogeneous group where the history, pathophysiology and epidemiology are diverse. In a recent review, orthostatic intolerance was perceived as a 'hidden danger' leading to syncopal attacks, increasing mortality and complicating the treatment of concomitant diseases such as hypertension and heart failure [39].

Finally, the risk for a syncope patient who drives can be measured as either the risk of recurrent syncope, or by the risk of recurrence while driving, or by the risk of recurrence while driving and with a related accident. This risk can then be compared with accident rate and injury rates of drivers currently allowed to drive. To date, it is unclear if syncope patients have increased risk of motor vehicle accidents and consequently it is unknown if the rate of motor vehicle accidents among syncope patients exceed the rate set as "tolerable" by society [40].

\section{Conclusions}

In conclusion, whether syncope is an independent risk marker in the general population really relates to the outcome in question. Syncope in general is related to a higher risk of subsequent falls and injury, and cardiac syncope is associated with increased mortality as compared to non-cardiac syncope. The overall prognosis in the general population, however, is by large determined by the underlying presence and severity of a given cardiac disease. A hospitalization for syncope even among healthy individuals is, however associated with increased mortality, perhaps due to unrecognized CVD. Moreover, there seems no doubt that syncope in general is a risk predictor of a recurrence (although this may differ by age, gender, and presumed cause of syncope). In the general population, it is important to recognize several risk factors associated with adverse outcome in order to safely navigate in a population where most patients with syncope are healthy and low-risk but where a small number of patients have life-threatening conditions. Further research in the general population should attempt to categorize which patients with syncope need immediate referral and diagnostic testing and whether this affects the outcome.

\section{Conflict of interest: None declared}




\section{References}

1. Ammirati F, Colivicchi F, Santini M. Diagnosing syncope in clinical practice. Implementation of a simplified diagnostic algorithm in a multicentre prospective trial: The OESIL 2 study (osservatorio epidemiologico della sincope nel lazio). Eur Heart J, 2000; 21: 935-940.

2. Sarasin FP, Louis-Simonet M, Carballo D et al. Prospective evaluation of patients with syncope: A population-based study. Am J Med, 2001; 111: 177-184.

3. Blanc JJ, L'Her C, Touiza A, Garo B, L'Her E, Mansourati J. Prospective evaluation and outcome of patients admitted for syncope over a 1 year period. Eur Heart J, 2002; 23: 815-820.

4. Disertori M, Brignole M, Menozzi C et al. Management of patients with syncope referred urgently to general hospitals. Europace, 2003; 5: 283-291.

5. Olde Nordkamp LR, van Dijk N, Ganzeboom KS et al. Syncope prevalence in the ed compared to general practice and population: A strong selection process. Am J Emerg Med, 2009; 27: 271-279.

6. Ruwald MH, Lock Hansen M, Lamberts M et al. Unexplained syncope and diagnostic yield of tests in syncope according to the ICD-10 discharge diagnosis. J Clin Med Res, 2013; 5: 441-450.

7. Ruwald MH, Hansen ML, Lamberts $M$ et al. The relation between age, sex, comorbidity, and pharmacotherapy and the risk of syncope: A Danish nationwide study. Europace, 2012; 14: 1506-1514.

8. Moya A, Sutton R, Ammirati F et al. Guidelines for the diagnosis and management of syncope (version 2009). Eur Heart J, 2009; 30: 2631-2671.

9. Soteriades ES, Evans JC, Larson MG et al. Incidence and prognosis of syncope. N Engl J Med, 2002; 347: 878-885.

10. Ganzeboom KS, Mairuhu G, Reitsma JB, Linzer M, Wieling W, van Dijk N. Lifetime cumulative incidence of syncope in the general population: A study of 549 dutch subjects aged 35-60 years. J Cardiovasc Electrophysiol, 2006; 17: 1172-1176.

11. Kapoor WN. Evaluation and outcome of patients with syncope. Medicine (Baltimore), 1990; 69: 160-175.

12. Kapoor WN, Hanusa BH. Is syncope a risk factor for poor outcomes? Comparison of patients with and without syncope. Am J Med, 1996; 100: 646-655.

13. Vanbrabant P, Gillet JB, Buntinx F, Bartholomeeusen S, Aertgeerts B. Incidence and outcome of first syncope in primary care: A retrospective cohort study. BMC Fam Pract, 2011; 12: 102.

14. Reed MJ, Newby DE, Coull AJ, Prescott RJ, Jacques KG, Gray AJ The rose (risk stratification of syncope in the emergency department) study. J Am Coll Cardiol, 2010; 55: 713-721.

15. Colivicchi F, Ammirati F, Melina D, Guido V, Imperoli G, Santini M. Development and prospective validation of a risk stratification system for patients with syncope in the emergency department: The OESIL risk score. Eur Heart J, 2003; 24: 811-819.

16. Quinn J, McDermott D, Stiell I, Kohn M, Wells G. Prospective validation of the san francisco syncope rule to predict patients with serious outcomes. Ann Emerg Med, 2006; 47: 448-454.

17. Quinn J, McDermott D, Kramer N et al. Death after emergency department visits for syncope: How common and can it be predicted? Ann Emerg Med, 2008; 51: 585-590.

18. Birnbaum A, Esses D, Bijur P, Wollowitz A, Gallagher EJ. Failure to validate the san francisco syncope rule in an independent emergency department population. Ann Emerg Med, 2008; 52: 151-159.

19. Del Rosso A, Ungar A, Maggi R et al. Clinical predictors of cardiac syncope at initial evaluation in patients referred urgently to a general hospital: The egsys score. Heart, 2008; 94: 1620-1626.

20. Martin TP, Hanusa BH, Kapoor WN. Risk stratification of patients with syncope. Ann Emerg Med. 1997; 29: 459-466.
21. Costantino G, Perego F, Dipaola F et al. Short- and long-term prognosis of syncope, risk factors, and role of hospital admission: Results from the steps (short-term prognosis of syncope) study. J Am Coll Cardiol, 2008; 51: 276-283.

22. Ungar A, Del Rosso A, Giada F et al. Early and late outcome of treated patients referred for syncope to emergency department: The egsys 2 follow-up study. Eur Heart J, 2010; 31: 2021-2026.

23. Reed MJ, Henderson SS, Newby DE, Gray AJ. One-year prognosis after syncope and the failure of the rose decision instrument to predict one-year adverse events. Ann Emerg Med, 2011; 58: 250-256.

24. Ruwald MH, Ruwald AC, Jons C et al. Evaluation of the chads2 risk score on short- and long-term all-cause and cardiovascular mortality after syncope. Clin Cardiol, 2013; 36: 262-268.

25. D'Ascenzo F, Biondi-Zoccai G, Reed MJ et al. Incidence, etiology and predictors of adverse outcomes in 43,315 patients presenting to the emergency department with syncope: An international meta-analysis. Int J Cardiol, 2013; 167: 57-62.

26. Numeroso F, Mossini G, Lippi G, Cervellin G. Evaluation of the current prognostic role of cardiogenic syncope. Intern Emerg Med, 2013; 8: 69-73.

27. Ruwald MH, Hansen ML, Lamberts M et al. Prognosis among healthy individuals discharged with a primary diagnosis of syncope. J Am Coll Cardiol, 2013; 61: 325-332.

28. van Dijk N, Boer KR, Colman N, Bakker A et al. High diagnostic yield and accuracy of history, physical examination, and ECG in patients with transient loss of consciousness in fast: The fainting assessment study. J Cardiovasc Electrophysiol, 2008; 19: 48-55.

29. Linzer M, Yang EH, Estes NA, 3rd, Wang P, Vorperian VR, Kapoor WN. Diagnosing syncope. Part 1: Value of history, physical examination, and electrocardiography. Clinical Efficacy Assessment Project of the American College of Physicians, 1997; 126: 989-996.

30. Olshansky B, Poole JE, Johnson G et al. Syncope predicts the outcome of cardiomyopathy patients: Analysis of the scd-heft study. J Am Coll Cardiol, 2008; 51: 1277-1282.

31. Ruwald MH, Okumura K, Kimura T et al. Syncope in high-risk cardiomyopathy patients with implantable defibrillators: Frequency, risk factors, mechanisms, and association with mortality: Results from the multicenter automatic defibrillator implantation trial-reduce inappropriate therapy (MADIT-RIT) study. Circulation, 2014; 129: 545-552.

32. Olshansky B, Sullivan RM. Sudden death risk in syncope: The role of the implantable cardioverter defibrillator. Prog Cardiovasc Dis, 2013; 55: 443-453.

33. Ruwald MH. Mechanism and prognosis of syncope in acquired and inherited cardiac disorders. J Cardiol Ther, 2014; 1: 17-26.

34. Barbic F, Angaroni L, Orlandi M et al. [syncope and work: Role of the occupational physician and global risk stratification]. G Ital Med Lav Ergon, 2011; 33: 303-305.

35. Barbic F, Dipaola F, Solbiati M, Furlan R. Do work accidents play any role in the increased risk of death observed in 25- to 44-year-old patients after syncope? J Am Coll Cardiol, 2013; 61: 2488-2489.

36. Sun BC, Costantino G, Barbic F et al. Priorities for emergency department syncope research. Ann Emerg Med, 2014; Published Online: May 29, 2014; DOI: http://dx.doi.org/10.1016/j. annemergmed.2014.04.014.

37. Ruwald MH, Hansen ML, Lamberts $\mathrm{M}$ et al. Comparison of incidence, predictors, and the impact of co-morbidity and polypharmacy on the risk of recurrent syncope in patients $<85$ versus $\geq 85$ years of age. Am J Cardiol, 2013; 112: 1610-1615.

38. Kenny RA, Bhangu J, King-Kallimanis BL. Epidemiology of syncope/collapse in younger and older western patient populations. Prog Cardiovasc Dis, 2013; 55: 357-363.

39. Fedorowski A, Melander O. Syndromes of orthostatic intolerance: A hidden danger. J Intern Med, 2013; 273: 322-335.

40. Sakaguchi S, Li H. Syncope and driving, flying and vocational concerns. Prog Cardiovasc Dis, 2013; 55: 454-463. 